\title{
A NOTE ON HYPERGEOMETRIC POLYNOMIALS
}

\author{
M. I. QURESHI and M. A. PATHAN ${ }^{1}$
}

(Received 16 May 1983; revised in October 1983)

\begin{abstract}
In a paper which appeared in this journal, Manocha and Sharma [6] obtained some results of Carlitz [4], Halim and Salam [5] and generalized a few of them by using fractional derivatives. The present paper is concerned with some erroneous results of this paper [6]. Many more sums of the product of hypergeometric polynomials are also obtained.
\end{abstract}

\section{Introduction}

Let $D_{x}^{b}$ be an operator of a fractional derivative having the order $b$, as usually defined by

$$
D_{x}^{b}\left[x^{a-1}\right]=\frac{d^{b}\left[x^{a-1}\right]}{d x^{b}}=\frac{\Gamma(a)}{\Gamma(a-b)} x^{a-b-1}
$$

which holds for all values of $b$ except $b=a$ and $a$ is neither zero nor a negative integer.

In our investigations, we shall use the following result which may be verified from (1.1) and the binomial theorem

$$
D_{x}^{c-a}\left[x^{m+c-1}(1-x)^{n}\right]=\frac{x^{(m+a-1)} \Gamma(c)(c)_{m}}{\Gamma(a)(a)_{m}} F_{1}\left[\begin{array}{l}
-n, c+m ; \\
a+m ;
\end{array}\right]
$$

where $m, n$ are non-negative integers, $|x|<1$ and ${ }_{2} F_{1}$ is Gauss's hypergeometric polynomial [8, page 45(1)]. Other elementary identities to be used are

$$
(a+1)_{m}=(-1)^{m}(-a-m)_{m} \text { and }(a+1)_{n}=(a+1)_{m}(a+m+1)_{n-m} .
$$

\footnotetext{
${ }^{1}$ Department of Mathematics, Aligarh Muslim University, Aligarh-202 001, India.

(c) Copyright Australian Mathematical Society 1984, Serial-fee code 0334-2700/84
} 
In an attempt to generalize some results of Carlitz [4] and Halim and Salam [5], Manocha and Sharma [6, page 475(30,32,34)] obtained the following results

$$
\begin{aligned}
& \sum_{n=0}^{\infty} \sum_{m=0}^{n} \frac{(c)_{m}(d)_{n-m} x^{m}(-y)^{n-m}}{(a)_{m}(b)_{n-m} m !(n-m) !} t_{2}^{n} F_{1}\left[\begin{array}{lr}
-(n-m), c+m ; \\
a+m ;
\end{array}\right] \\
& \quad \times{ }_{2} F_{1}\left[\begin{array}{lr}
-m, d+n-m ; & y \\
b+n-m ; &
\end{array}\right]=\sum_{n=0}^{\infty} t^{n}{ }_{3} F_{2}\left[\begin{array}{lr}
-n, c, 1-b-n ; \\
a, 1-d-n ;
\end{array}\right],(1.4) \\
& { }_{3} F_{2}\left[\begin{array}{lr}
-n, c, b-d ; & 1 \\
1+c-a-n, 1-d-n ;
\end{array}\right]=\frac{(c)_{n}}{(a-c)_{n}} F_{2}\left[\begin{array}{ll}
-n, c, 1-b-n ; & 1 \\
a, 1-d-n ; & 1
\end{array}\right]
\end{aligned}
$$

and

$$
\sum_{m=0}^{n} \frac{(-b-n)_{m}}{(1+a)_{m}} L_{m}^{(a)}(x) L_{n-m}^{(b)}(-x)=\frac{x^{n}(1+a+b)_{2 n}}{(1+a)_{n}(1+b)_{n}(1+a+b)_{n}},
$$

respectively. Here ${ }_{3} F_{2}$ and $L_{m}^{(a)}(x)$ are generalized hypergeometric function [8, page 73(2)] and associated Laguerre polynomial [8, page 200(1)] respectively.

It does not appear to have been observed previously that (1.4) to (1.6) are not correct, although many workers have used them lately.

By making use of fractional derivatives, we give corrections to results (1.4) and (1.5) in Section 2. Later in Section 3, we apply manipulations of series to get summation for product of hypergeometric polynomials which are further specialized in terms of Jacobi [8, page 254(1)] and Laguerre type polynomials. One of the special cases of these results yields the correct form of the result (1.6).

\section{Use of fractional derivatives}

Consider an elementary result

$$
e^{x(1-y) t} e^{-y(1-x) t}=e^{x t} e^{-y t},
$$

which may be written in the form

$$
\sum_{m, n=0}^{\infty} \frac{(-1)^{n} x^{m}(1-y)^{m} y^{n}(1-x)^{n} t^{m+n}}{m ! n !}=\sum_{m, n=0}^{\infty} \frac{(-1)^{n} x^{m} y^{n} t^{m+n}}{m ! n !} .
$$


Now multiplying both sides of (2.2) by $x^{c-1} y^{d-1}$, applying the operators $D_{x}^{c-a}, D_{y}^{d-b}$ on both sides and using the results (1.1) and (1.2), we get

$$
\begin{aligned}
& \sum_{n=0}^{\infty} \sum_{m=0}^{n} \frac{(c)_{m}(d)_{n-m} x^{m}(-y)^{n-m}}{(a)_{m}(b)_{n-m} m !(n-m) !} t_{2}^{n} F_{1}\left[\begin{array}{ll}
-(n-m), c+m ; & x \\
a+m ; &
\end{array}\right] \\
& \times_{2} F_{1}\left[\begin{array}{l}
-m, d+n-m ; \\
b+n-m ;
\end{array}\right]=\sum_{n=0}^{\infty} \frac{t^{n}(-y)^{n}(d)_{n}}{(b)_{n} n !}{ }_{3} F_{2}\left[\begin{array}{ll}
-n, c, 1-b-n ; & x / y \\
a, 1-d-n ; &
\end{array}\right]
\end{aligned}
$$

which is the correct form of the result (1.4). The equation (2.3) requires that $a, b, c, d$ are not zero or negative integers.

We now turn to an error in equation (1.5). On equating the coefficients of $t^{n}$ on both the sides in (2.3), we get

$$
\begin{aligned}
\sum_{m=0}^{n}\left(\begin{array}{c}
n \\
m
\end{array}\right) & \frac{(c)_{m}(1-b-n)_{m}}{(a)_{m}(1-d-n)_{m}}\left(\frac{-x}{y}\right)^{m}{ }_{2} F_{1}\left[\begin{array}{ll}
-(n-m), c+m ; & \\
a+m ;
\end{array}\right] \\
& \times{ }_{2} F_{1}\left[\begin{array}{lr}
-m, d+n-m ; \\
b+n-m ;
\end{array}\right]={ }_{3} F_{2}\left[\begin{array}{ll}
-n, c, 1-b-n ; & x / y \\
a, 1-d-n ; &
\end{array}\right],
\end{aligned}
$$

which is the result of Manocha and Sharma [6, page 475(31)].

Putting $x=y=1$ in (2.4), we get

$$
{ }_{3} F_{2}\left[\begin{array}{ll}
-n, c, b-d ; & 1 \\
1-d-n, 1-a-n+c ; & 1
\end{array}\right]=\frac{(a)_{n}}{(a-c)_{n}}{ }_{3} F_{2}\left[\begin{array}{ll}
-n, c, 1-b-n ; & 1 \\
a, 1-d-n ; & 1
\end{array}\right],
$$

which is the correct form of equation (1.5).

In fact equation (2.5) is a well known result of Bailey [3, page 238(2.1)] (see also [1, page 10(26)]) but Abiodun [1, page 10] has stated that the result (2.5) is due to Whipple [10]. However, we have not been able to find it in [10]. In fact the result of Whipple [10, page 105(2)] (see also [2, page 14(1)] and [9, equation 11]) is

$$
{ }_{3} F_{2}\left[\begin{array}{ll}
a, b, c ; & \\
e, f ; & 1
\end{array}\right]=\frac{\Gamma(e) \Gamma(f) \Gamma(s)}{\Gamma(a) \Gamma(s+b) \Gamma(s+c)}{ }_{3} F_{2}\left[\begin{array}{ll}
e-a, f-a, s ; & \\
s+b, s+c ; & 1
\end{array}\right]
$$


where $s=e+f-a-b-c, \operatorname{Re}(a)>0$ and $\operatorname{Re}(s)>0$, which clearly differs from the result (2.5).

Now replacing $x, y, a, b, c$ and $m$ in (2.4) by $x / c, y / d, 1+a, 1+b,-d$ and $n-m$ respectively, and making $d \rightarrow \infty$, we get

$$
\begin{aligned}
n !\left(\frac{x}{y}\right)^{n} \sum_{m=0}^{n} \frac{(-b-n)_{n-m}}{(1+a)_{n-m} m !(n-m) !}\left(\frac{y}{x}\right)^{m}{ }_{1} F_{1}\left[\begin{array}{ll}
-m ; & x \\
1+a+n-m ;
\end{array}\right] \\
\times{ }_{1} F_{1}\left[\begin{array}{ll}
-(n-m) ; \\
1+b+m ;
\end{array}\right]={ }_{2} F_{1}\left[\begin{array}{ll}
-n,-b-n ; & -\frac{x}{y} \\
1+a ; &
\end{array}\right],
\end{aligned}
$$

which can be put in the form of

$$
\sum_{m=0}^{n}\left(\frac{-y}{x}\right)^{m} L_{m}^{(a+n-m)}(x) L_{n-m}^{(b+m)}(y)=\left(\frac{x+y}{x}\right)^{n}(-1)^{n} P_{n}^{(a, b)}\left(\frac{y-x}{y+x}\right)
$$

by using the results for Laguerre and Jacobi polynomials [8, pages 200(1), 254(2)].

Setting $y=-x$ in (2.7), using Gauss's summation theorem [8, page 69(4)] and [8, page 200(1)], we get an interesting result, in which a finite sum of the product of two generalized Laguerre polynomials having the same argument but opposite sign, different order and degree, is equal to a constant for every value of the argument, that is,

$$
\sum_{m=0}^{n} L_{m}^{(a+n-m)}(x) L_{n-m}^{(b+m)}(-x)=(1+a+b+n)_{n} / n !
$$

\section{Use of series manipulations}

In this section some more finite summations of the products of two hypergeometric polynomials are obtained by using the method of series manipulations.

Consider the series,

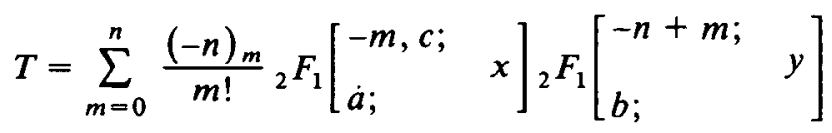


which can be put in the following forms,

$$
\begin{aligned}
T & =\sum_{m=0}^{n} \sum_{r=0}^{m} \sum_{s=0}^{n-m} \frac{(-n)_{m}(-m)_{r}(c)_{r}(m-n)_{s}(d)_{s}}{(a)_{r}(b)_{s} m !} \frac{x^{r} y^{s}}{r ! s !} \\
& =\sum_{m=0}^{n} \sum_{r=0}^{m} \sum_{s=0}^{n-m} \frac{(-1)^{m}(c)_{r}(d)_{s} n !(-x)^{r}(-y)^{s}}{(a)_{r}(b)_{s} r ! s !(m-r) !(n-m-s) !} \\
& =\sum_{s=0}^{n} \sum_{r=0}^{n-s} \sum_{m=0}^{n-r-s} \frac{(-1)^{m}(c)_{r}(d)_{s} n ! x^{r}(-y)^{s}}{(a)_{r}(b)_{s} r ! s ! m !(n-m-r-s) !} \\
& =\sum_{s=0}^{n} \sum_{r=0}^{n-s} \frac{n !(c)_{r}(d)_{s} x^{r}(-y)^{s}}{(a)_{r}(b)_{s} r ! s !(n-r-s) !} \sum_{m=0}^{n-r-s}(-1)^{m}\left(\begin{array}{c}
n-r-s \\
m
\end{array}\right) \\
& =n ! \sum_{s=0}^{n} \frac{(d)_{s}(-y)^{s}}{(b)_{s} s !} \sum_{r=0}^{n-s} \frac{(c)_{r} x^{r}}{(a)_{r} r !(n-r-s) !} \sum_{m=0}^{n-r-s}(-1)^{m}\left(\begin{array}{c}
n-r-s \\
m
\end{array}\right),
\end{aligned}
$$

where $\left(\begin{array}{c}n-r-s \\ m\end{array}\right)$ is a binomial coefficient.

Now we observe that the value of the following series

$$
A_{r}=\sum_{r=0}^{n-s} \frac{(c)_{r} x^{r}}{(a)_{r} r !(n-r-s) !} \sum_{m=0}^{n-r-s}(-1)^{m}\left(\begin{array}{c}
n-r-s \\
m
\end{array}\right)
$$

will be zero for $r=0,1,2, \ldots, n-s-1$ and thus for $r=(n-s)$, we get

$$
A_{n-s}=\frac{(c)_{n-s} x^{n-s}}{(a)_{n-s}(n-s) !}=\frac{(c)_{n}(-n)_{s}(1-a-n)_{s} x^{n}}{(a)_{n}(1-c-n)_{s} n !}\left(\frac{-1}{x}\right)^{s} \text {. }
$$

Therefore from (3.1), (3.2) with (3.3) and (3.4), we have

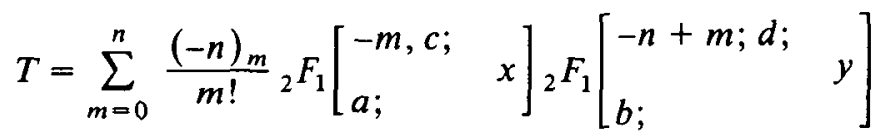

$$
\begin{aligned}
& =\frac{(c)_{n} x^{n}}{(a)_{n}}{ }_{3} F_{2}\left[\begin{array}{l}
-n, 1-a-n, d ; \\
b, 1-c-n ;
\end{array}\right]
\end{aligned}
$$

which was obtained by Manocha and Sharma [7, page 233(15)] by using the method of fractional derivatives.

When $x=y=1$, equation (3.5) reduces to a transformation of ${ }_{3} F_{2}$ having the unit argument, ${ }_{3} F_{2}\left[\begin{array}{ll}-n, a-c, 1-b-n ; & 1 \\ a, 1-b-n+d ; & 1\end{array}\right]=\frac{(b)_{n}(c)_{n}}{(b-d)_{n}(a)_{n}}{ }_{3} F_{2}\left[\begin{array}{ll}-n, d, 1-a-n ; & \\ b, 1-c-n ; & 1\end{array}\right]$. 
Now replacing $x, y, a, b$ and $c$ in (3.5) by $x / c, y / d, 1+a, 1+b$ and $-d$, respectively, and making $d \rightarrow \infty$, we get

$$
\begin{aligned}
& \sum_{m=0}^{n} \frac{(-n)_{m}}{m !}{ }_{1} F_{1}\left[\begin{array}{l}
-m ; \\
1+a ;
\end{array}\right]{ }_{1} F_{1}\left[\begin{array}{ll}
-n+m ; \\
1+b ;
\end{array}\right] \\
& =\frac{x^{n}}{(1+a)_{n}}{ }_{2} F_{1}\left[\begin{array}{ll}
-n,-a-n ; & -y / x \\
1+b ; &
\end{array}\right],
\end{aligned}
$$

which reduces to a known result of Manocha and Sharma [6, page 475(33)],

$$
\sum_{m=0}^{n} \frac{(-b-n)_{m}}{(1+a)_{m}} L_{m}^{(a)}(x) L_{n-m}^{(b)}(y)=\frac{(-1)^{n}(x+y)^{n}}{(1+a)_{n}} P_{n}^{(a, b)}\left(\frac{y-x}{y+x}\right),
$$

by using the results $[8$, pages $200(1), 255(8)]$.

Setting $y=-x$ in (3.7), we get after manipulations

$$
\sum_{m=0}^{n} \frac{(-b-n)_{m}}{(1+a)_{m}} L_{m}^{(a)}(x) L_{n-m}^{(b)}(-x)=\frac{(1+a+b+n)_{n} x^{n}}{(1+a)_{n} n !}
$$

which is the correct form of equation (1.6).

Furthermore Manocha and Sharma [6] have stated that equations (3.8) and (3.9) are confluent cases of [6, page $475(31)]$ i.e., equation (2.4). It may be observed that these equations are not confluent cases of [6, page 475(31)]. In fact the confluent cases of [6, page 475(31)] are equations (2.8) and (2.9).

\section{Acknowledgement}

The authors are thankful to the referees for their valuable suggestions.

\section{References}

[1] R. F. A. Abiodun, "Transformation formulae for Kampé de Fériet function", Nederl. Akad. Wetensch. Proc. Ser. A 83 (1980), 1-11.

[2] W. N. Bailey, Generalized hypergeometric series (Cambridge University Press, London, 1935).

[3] W. N. Bailey, "On the sum of a terminating ${ }_{3} F_{3}(1)$ ", Quart. J. Math. Oxford Ser. (2) 4 (1953), 237-240.

[4] L. Carlitz, "A note on the Laguerre polynomials", Michigan Math. J. 7 (1960), 219-223.

[5] N. A. Halim and W. A. Al-Salam, "Double Euler transformations of certain hypergeometric functions", Duke Math. J. 30 (1963), 51-62. 
[6] H. L. Manocha and B. L. Sharma, "Summation of infinite series", J. Austral. Math. Soc. 6 (1966), 470-476.

[7] H. L. Manocha and B. L. Sharma, "Some formulae by means of fractional derivatives", Compositio Math. 18(3) (1967), 229-234.

[8] E. D. Rainville, Special functions (Macmillan, New York, 1960).

[9] J. Thomas, "Üeber die Funktionen welche durch Reihen von der Form dargestellt werden, $1+p p^{\prime} p^{\prime \prime} / 1 q^{\prime} q^{\prime \prime}+\cdots ", J$. Reine Angew. Math. 87 (1879), 26-73.

[10] F. J. W. Whipple, "A group of generalized hypergeometric series relations between 120 allied series of the type $F[a, b, c ; d, e]$ ", Proc. London Math. Soc. (2) 23 (1925), 104-114. 